\title{
PREVENCIÓN DE LA MUCOSITIS ORAL SECUNDARIA A TRATAMIENTOS ONCOLÓGICOS EN ADULTOS CON CÁNCER: REVISIÓN SISTEMÁTICA
}

\author{
${ }^{1}$ Johanna Alejandra Otero Wandurraga, ${ }^{2}$ Harold Torres Pinzón, ${ }^{3}$ Ana María Pedraza Flechas, ${ }^{3}$ Abel Ernesto González Vélez. \\ ${ }^{1}$ Odontóloga U. Santo Tomás, Especialista en Epidemiología U. El Bosque, Epidemiologa Observatorio de Salud Pública de Santander, Bucaramanga (Colombia). \\ ${ }^{2}$ Odontólogo U. Santo Tomás, Especialista en Epidemiología U. El Bosque, Bogotá (Colombia). \\ ${ }^{3}$ Médico, Especialista en Epidemiología U. El Bosque. Candidato a Magíster en Salud Pública Escuela Nacional de Sanidad, Instituto Carlos III, Madrid (España).
}

Autor responsable de correspondencia: Johanna Alejandra Otero W. Correo electrónico: jaoterow@gmail.com

\begin{abstract}
Resumen
Objetivo: Evaluar la efectividad clínica de intervenciones preventivas sistémicas o tópicas para la mucositis oral secundaria a tratamientos antineoplásicos en adultos con cáncer.

Materiales y métodos: Se realizaron búsquedas en Cochrane, MEDLINE, LILACS y SCIELO. Fueron seleccionados ensayos clínicos controlados aleatorizados. Las medidas preventivas debían ser iniciadas el mismo día del inicio del tratamiento antineoplásico. Se incluyeron límites de tiempo en la búsqueda. Inicialmente se revisaron título y resumen de todos los estudios resultantes de la búsqueda y posteriormente en texto completo, la extracción de resultados se realizó por duplicado y las diferencias fueron resueltas por discusión. Se hizó un metanálisis con los estudios que fueron comparables en los programas EPIDAT y REVMAN 5.

Resultados: Cuarenta y seis estudios fueron elegibles de los cuales se excluyeron 24. Se evaluaron 15 intervenciones preventivas, de ellas, nueve presentaron algún beneficio, tres intervenciones se presentaron en más de un estudio y fueron comparables para la realización de metanálisis: el amifostine, láser He/Ne y zinc. El zinc fue el único que mostró beneficio; el riesgo de desarrollar mucositis grado 2 en los pacientes que recibieron zinc fue $20 \%$ menor que en el grupo control y $73 \%$ menor para desarrollar mucositis grado 3 . Conclusiones: La administración del zinc, la miel y el sucralfate han mostrado algún beneficio en la prevención o la reducción de la severidad de la mucositis asociada con el tratamiento del cáncer. La calidad y el tamaño de la muestra de estos estudios no son suficientes para recomendar estas intervenciones específicas. [Otero JA, Torres H, Pedraza AM, González AE. Prevención de la mucositis oral secundaria a tratamientos oncológicos en adultos con cáncer: revisión sistemática. Ustasalud 2011; 10: 11 - 28]
\end{abstract}

Palabras clave: Mucositis, Neoplasmas, Ensayos clínicos aleatorizados, Estomatitis, Sucralfate.

\section{PREVENTION OF ORAL MUCOSITIS IN CANCER PATIENTS WITH ANTINEOPLASIC TREATMENT:}

\section{A SYSTEMATIC REVIEW}

\begin{abstract}
Objective: To evaluate the effectiveness of interventions for the prevention of oral mucositis in cancer patients antineoplasic treated. Methods: The Cochrane Oral Health Group Trials Register, MEDLINE LILACS y SCIELO were searched. Randomized clinical trials were selected. The preventive measures must have been begun the first day of the antineoplasic treatment. Only were include the studies publish in the last 10 years. Review the title and abstract of each result of the search and extracted the outcome measures and results in duplicate. Meta-analysis was performed when two or more studies use the same intervention and was comparable. The statistical analysis was made by EPIDAT y RevMan 5.

Results: Forty six studies were eligible, 24 were excluded. Fifteen interventions were evaluated, of the included interventions in this study nine showed evidence of some benefit for either preventing or reducing the severity of mucositis, three interventions were present in more than one study and comparable for performing meta-analysis: amifostine, laser $\mathrm{He} / \mathrm{Ne}$ y zinc. The meta-analysis of zinc was the only one that showed beneficial effect in preventing moderate and severe mucositis.

Conclusions: Especially administration of zinc, honey and sucralfate had showed some benefit at preventing or reducing the severity of mucositis associated with cancer treatment. Nevertheless the quality and the sample size of these studies are not enough for recommend and specific intervention instead of another.
\end{abstract}

Key words: Mucositis, Neoplasms head and neck, Randomized controlled trials, Stomatitis, Sucralfate. 


\section{INTRODUCCIÓN}

La quimioterapia y la radioterapia, son tratamientos con efectos citotóxicos que atacan tejidos anormales y normales con alta tasa de recambio como los de la mucosa oral, el epitelio del tracto gastrointestinal y la medula ósea. ${ }^{1,2}$

Las complicaciones orales más frecuentes asociadas al tratamiento del cáncer son: estomatitis, infección, sangrado, mucositis, dolor, pérdida de la función y xerostomía..$^{3-5}$ La limitación más importante producida por la mucositis oral es el dolor. ${ }^{6}$ Se ha estimado que la mucositis oral secundaria a quimioterapia es menor que la mucositis segundaria a radioterapia, su incidencia varía de acuerdo con las dosis de administración. ${ }^{4,7,8}$

La mucositis oral se divide en cuatro fases: inflamatoria vascular, epitelial, ulcerativa microbiológica y de remisión. La fase inicial se caracteriza por liberación de IL 1 y TNF, la fase epitelial produce liberación de agentes citotóxicos, con pérdida de las barreras de defensa la fase ulcerativa y por último en la fase de remisión hay una nueva proliferación celular y recuperación inmunológica. ${ }^{9}$

Las recomendaciones actuales presentan pruebas débiles y poco fiables de sus beneficios. Esta revisión sistemática de la literatura con metanálisis, tiene por objetivo sugerir cuál es la intervención más efectiva para la prevención de la mucositis oral en adultos con tumores malignos localizados en cabeza y cuello que requieren tratamiento de quimioterapia, radioterapia o ambas.

\section{MATERIALES Y MÉTODOS}

\section{Criterios para la selección de los estudios de esta revisión}

Tipo de estudios: Ensayos clínicos controlados aleatorizados que evalúen medidas preventivas de la mucositis oral secundaria.

Tipo de participantes: Estudios donde los participantes fueran adultos con mucositis oral aguda secundaria a tratamiento con quimioterapia, radioterapia o ambas con cáncer de cabeza y cuello.

Tipo de intervención: Manejo preventivo sistémico o local para la mucositis oral secundaria, iniciado el mismo día de tratamiento de quimioterapia, radioterapia o ambas a adultos con cáncer de cabeza y cuello.

Tipo de medidas de resultado: Desenlace primario: Tasa de curación de la mucositis oral se evaluó de acuerdo con la incidencia de mucositis grado 3 o mayor según los criterios RTOG. Desenlace secundario: Media del grado de mucositis al finalizar el tratamiento de radioterapia, quimioterapia o ambas.

\section{Estrategia de búsqueda para la identifica- ción de los estudios}

Bases de datos: Por medio de términos controlados se hizo una búsqueda sistemática electrónica en las siguientes bases de datos:

- Pub Med/MEDLINE: Base de datos de literatura biomédica internacional de la Biblioteca CENTRAL: Registro central de ensayos clínicos controlados del grupo Cochrane de la biblioteca nacional médica de los Estados Unidos.

- LILACS: Literatura Latinoamericana y del Caribe en ciencias de la salud.

- SCIELO: Biblioteca Científica Electrónica en Línea.

El periodo de búsqueda incluyó las publicaciones de la literatura científica de los últimos 10 años.

Términos de búsqueda: Este trabajo contó con una revisión inicial de literatura publicada y a partir de las palabras clave se definieron los términos generales de búsqueda, a continuación se definieron los términos secundarios de búsqueda a partir de los objetivos planteados para la revisión sistemática, cada término se buscó en el Thesaurus de PubMed para los MeSH (Medical SubHeading) y en los Descriptores de Ciencias de la Salud (DeCS) para LILACS y SCIELO. Se realizaron combinaciones de los términos que describen el tema central de investigación, y los términos restantes que muestran los tópicos específicos a analizar (Tabla 1).

\section{Métodos de la revisión}

Captura de artículos: Una vez obtenidas las referencias mediante las estrategias de búsqueda propuestas, se utilizó el software JabRef Reference Manager versión 2.4.2 para depurar la base de datos e identificar las citas duplicadas; se evaluaron de forma pareada los títulos y resúmenes para seleccionar los artículos que cumplieron los criterios de inclusión. A continuación se obtuvieron los textos completos de las citas potencialmente útiles para determinar si cumplían o no con estos criterios. 


\section{ARTÍCULO ORIGINAL}

Tabla 1. Términos y resultados de búsqueda en las bases de datos.

Pub Med/MEDLINE

No. de artículos

\#1. "Mucositis" [MeSH

4.790

\#2. "Stomatitis" [MeSH]

18.770

\#3. "head and neck neoplasms" [MeSH]

1.097 .286

\#4. “neoplasms” [MeSH]

2.062 .035

\#5. "Randomized controlled trial" [MeSH]

324.846

\#6. "Randomized controlled trial as topic" [MeSH]

8.709

\#7. \#1 OR \#2

22.359

\#8. \#3 OR \#4

2.062.035

\#9. \#5 OR \#6

324.846

\#11. \#7 AND \#8 AND \#9

705

\#12. Search (\#7 AND \#8 AND \#9) Limits: published in the last 10 years

389

\section{CENTRAL}

\#1. Neoplasms [MeSH]

33.274

\#2. Head and Neck Neoplasms [MeSH]

1.516

\#3. Stomatitis [MeSH]

1.143

\#4. Mucositis [MeSH]

944

\#5. \#1 OR \#2

33.274

\#6. \#3 OR \#4

1.806

\#7. \#5 AND \#6

781

\#8. \#7, from 1999 to 2009

348

\section{LILACS}

Mucositis OR Stomatitis and Neoplasms OR Head and Neck Neoplasms

45

SCIELO

Mucositis OR Stomatitis and Neoplasms OR Head and Neck Neoplasms

45

Fecha de búsqueda: Abril 16 de 2009.

Al menos dos revisores evaluaron la calidad de los estudios por medio de las recomendaciones del Manual del Revisor Cochrane versión 4.1.6 mediante los siguientes criterios: ocultamiento de la asignación del tratamiento, cegamiento de los pacientes, proveedores y evaluadores de los resultados, información sobre los motivos de retiro por grupos de intervención y porcentaje de retiros.

Evaluación de la validez: la validez de los estudios fue evaluada por los criterios propuestos por Cochrane, para esta revisión se tuvieron en cuenta los tres primeros criterios sin incluir el porcentaje de retiros (Tabla 2).

Tabla 2. Criterios individuales para evaluación de calidad de los estudios.

\begin{tabular}{ll}
\hline \multicolumn{1}{c}{ Criterios } & \multicolumn{1}{c}{ Valores } \\
\hline & A: Adecuado \\
Ocultamiento de la asignación del tratamiento & B: Poco claro \\
& C: Inadecuado \\
& D: No se usó ocultación \\
Cegamiento de los pacientes proveedores y evaluadores & Cumple \\
\hline Motivos de retiro & No cumple \\
& Inadecuado \\
\hline
\end{tabular}

Fuente: Manual de Revisores Cochrane. 
Se consideró el riesgo de sesgo en cada ensayo. Bajo riesgo, si había ocultación adecuada de la asignación, pacientes, proveedores y evaluación cegados e información sobre el motivo de los retiros. Moderado riesgo, de sesgo si se cumplía de forma poco clara y/o inadecuada con uno o más de los anteriores criterios y alto riesgo, cuando no se cumplía con uno o más de los criterios.

\section{Síntesis de datos}

Se extrajeron por separado los datos de los estudios seleccionados por medio de una tabla diseñada para tal fin. Ésta incluyó variables relacionadas con el país de realización del ensayo, tiempo de seguimiento de los participantes, número total de participantes, características clínicas de éstos, tipo de radioterapia y/o quimioterapia recibida, intervención de prevención comparada y característica de las medidas de resultado.
Se realizó metanálisis solamente con aquellos estudios que compararon las mismas estrategias de prevención y permitieron igualar sus medidas de resultado. Se realizó test de heterogeneidad de Cochrane y se cuantificó con el estadístico $\mathrm{I}^{2}$. En el caso de estudios homogéneos se utilizó el modelo de efectos fijos, mientras en estudios heterogéneos se prefirió el modelo de efectos aleatorios. El análisis estadístico se ejecutó por medio de los programas RevMan 5 y Epidat 3.1.

\section{RESULTADOS}

\section{Descripción de los estudios}

Características de los ensayos: se obtuvieron 489 citas como resultado de la estrategia de búsqueda descrita para las cuatro bases de datos señaladas: 46 y 44 estudios que cumplían con los criterios de selección de los artículos. Se revisaron en texto completo los estudios seleccionados por títulos y resúmenes, incluyéndose 22 ensayos (Tabla 3 )

Tabla 3. Características de los estudios incluidos en la revisión.

\begin{tabular}{|c|c|}
\hline Estudio & Antonadou 2002 \\
\hline Métodos & $\begin{array}{l}\text { Ensayo clínico controlado aleatorizado, grupos paralelos, desarrollado en Grecia. Pacientes, } \\
\text { proveedores y evaluadores no cegados. Se describen los motivos de las perdidas, } 10 \% \text {. } \\
\text { Seguimiento entre enero } 1997 \text { y enero 1998. } \mathrm{N}=50\end{array}$ \\
\hline Participantes & Adultos con carcinoma escamocelular de la cabeza y el cuello $\geq$ T2NOMO, sin metástasis \\
\hline $\begin{array}{l}\text { Régimen } \\
\text { quimioterapia - radioterapia }\end{array}$ & $\begin{array}{l}\text { Radioterapia convencional con rayos gamma de } 60 \mathrm{Co} \text {, dosis total entre } 60 \text { y } 74 \mathrm{~Gy} / 6-7,5 \\
\text { semana. Quimioterapia con carboplatino a dosis de } 90 \mathrm{mg} / \mathrm{m}^{2} / \text { semana antes de la radioterapia }\end{array}$ \\
\hline Intervenciones & $\begin{array}{l}\text { Grupo de estudio: Radioquimioterapia + Amifostine } \\
\text { Grupo control: Radioquimioterapia sola. Amifostine } 300 \mathrm{mg} / \mathrm{m}^{2} / \text { día IV } 15-30 \mathrm{~min} \text { antes de la } \\
\text { radioterapia }\end{array}$ \\
\hline Resultados & $\begin{array}{l}\text { Incidencia de mucositis aguda } \geq \text { grado } 3 \text { a la } 7^{\circ} \text { semana de radioquimioterapia. Evaluación por } \\
\text { medio de la escala del Radiation Therapy Oncology Group }\end{array}$ \\
\hline Ocultación & A - Adecuada \\
\hline Calidad & $\mathrm{C}$ - Alto riesgo de sesgo \\
\hline Estudio & Bourhis 2000 \\
\hline Métodos & $\begin{array}{l}\text { Ensayo clínico controlado aleatorizado, grupos paralelos, desarrollado en Francia. Pacientes, } \\
\text { proveedores y evaluadores no cegados. Se describen los motivos de las pérdidas, } 7,7 \% \text {. } \\
\text { Seguimiento entre mayo } 1996 \text { y febrero } 1998 . \mathrm{N}=26\end{array}$ \\
\hline Participantes & $\begin{array}{l}\text { Adultos con carcinoma escamocelular de orofaringe, cavidad oral, hipofaringe o laringe, y en } \\
\text { todos los casos con estadio IV no metastásico (UICC) }\end{array}$ \\
\hline $\begin{array}{l}\text { Régimen } \\
\text { quimioterapia - radioterapia }\end{array}$ & $\begin{array}{l}\text { Radioterapia fraccionada acelerada con rayos gamma } 60 \mathrm{Co} 1,25 \mathrm{MeV} \text { unidades. Dosis total de } \\
64 \mathrm{~Gy} \text { en } 3,5 \text { semanas }\end{array}$ \\
\hline Intervenciones & $\begin{array}{l}\text { Grupo de estudio: Radioterapia + Amifostine } \\
\text { Grupo control: Radioterapia. Amifostine } 300 \mathrm{mg} / \mathrm{m}^{2} / \text { día IV } 15-30 \mathrm{~min} \text { antes de la radioterapia }\end{array}$ \\
\hline Resultados & $\begin{array}{l}\text { Duración media e incidencia de mucositis aguda } \geq \text { grado } 3 \text { por medio de las escalas de la } \\
\text { Organización Mundial de la Salud y del Radiation Therapy Oncology Group durante 3,5 semanas } \\
\text { de radioterapia. }\end{array}$ \\
\hline Ocultación & A - Adecuada \\
\hline Calidad & $\mathrm{C}$ - Alto riesgo de sesgo \\
\hline
\end{tabular}


Estudio

Métodos

Participantes

Régimen quimioterapia radioterapia

Intervenciones

Resultados

Ocultación

Calidad

\section{Brizel 2000}

Ensayo clínico controlado aleatorizado, grupos paralelos, multicéntrico. Pacientes, proveedores y evaluadores no cegados. Se describen los motivos de las pérdidas, $22,8 \%$. Seguimiento entre octubre 1995 y octubre de 1997. $\mathrm{N}=315$

Adultos con carcinoma escamocelular de cabeza y cuello. $84 \%$ de los pacientes con estadio mayor o igual a T2

Radioterapia fraccionada estándar, dosis total: 50-70Gy

Grupo de estudio: Radioterapia + Amifostine

Grupo control: Radioterapia sola. Amifostine $200 \mathrm{mg} / \mathrm{m}^{2} / \mathrm{d}$ IV $15-30 \mathrm{~min}$. antes de la radioterapia

Incidencia de mucositis aguda (90 días) $\geq$ grado 3 evaluada semanalmente por medio de la escala del Radiation Therapy Oncology Group

A - Adecuada

$\mathrm{C}$ - Alto riesgo de sesgo
Estudio

Métodos

Participantes

Régimen quimioterapia radioterapia

Intervenciones

Resultados

Ocultación

Calidad

Estudio

\section{Buentzel 2006}

Ensayo clínico controlado aleatorizado, grupos paralelos, multicéntrico. Pacientes, proveedores y evaluadores cegados. Se describen los motivos de las perdidas, 22,7\%. Seguimiento entre octubre 1996 y noviembre de $2000 . \mathrm{N}=132$

Adultos con carcinoma escamocelular de cabeza y cuello. 90\% de los participantes con estadio T2 o mayor

Radioterapia fraccionada estándar, dosis total 60-70Gy/6-7 semana. Los días 1 a 5 y 21 a 25 quimioterapia con carboplatino $70 \mathrm{mg} / \mathrm{m}^{2}$ IV $30 \mathrm{~min}$. después del amifostine

Grupo de estudio: Carboplatino + radioterapia + Amifostine

Grupo control: Carboplatino + Radioterapia + placebo

Días 1-5 y 21-25: Amifostine $300 \mathrm{mg} / \mathrm{m}^{2} / \mathrm{IV}$

Días 6-20 y 26-30/35: Amifostine $200 \mathrm{mg} / \mathrm{m}^{2}$ IV

Incidencia de mucositis aguda (90 días) $\geq$ grado 3 evaluada semanalmente por medio de la escala del Radiation Therapy Oncology Group

A - Adecuada

A - Bajo riesgo de sesgo

\begin{tabular}{|c|c|}
\hline Estudio & Ertekin 2004 \\
\hline Métodos & $\begin{array}{l}\text { Ensayo clínico controlado aleatorizado, grupos paralelos, desarrollado en Turquía. Pacientes y } \\
\text { proveedores cegados. Se describen los motivos de las perdidas, } 10 \% \text {. Seguimiento entre mayo } \\
2001 \text { y mayo } 2002 . \mathrm{N}=30\end{array}$ \\
\hline Participantes & $\begin{array}{l}\text { Adultos con cáncer de cabeza y cuello, } 3 \text { participantes en cada brazo recibieron quimioterapia } \\
\text { concurrente }\end{array}$ \\
\hline $\begin{array}{l}\text { Régimen quimioterapia - } \\
\text { radioterapia }\end{array}$ & $\begin{array}{l}\text { Radioterapia convencional fraccionada con telecobalto, dosis: } 2 \text { Gy/fracción, } 5 \text { fracciones/ } \\
\text { semana/4-7semanas, dosis media total: } 64 \mathrm{~Gy}\end{array}$ \\
\hline Intervenciones & $\begin{array}{l}\text { Grupo de estudio: Radioterapia + Zinc } \\
\text { Grupo control: radioterapia + placebo } \\
\text { Zinc cápsulas 50mg tres veces al día desde el inicio de la RT hasta } 6 \text { semanas después de la } \\
\text { radioterapia }\end{array}$ \\
\hline Resultados & $\begin{array}{l}\text { Evaluación del grado de mucositis oral semanalmente por medio de la escala del } \\
\text { Radiation Therapy Oncology Group }\end{array}$ \\
\hline Ocultación & A - Adecuada \\
\hline Calidad & A - Bajo riesgo de sesgo \\
\hline
\end{tabular}


Estudio

Métodos

Participantes

Régimen quimioterapia radioterapia

Intervenciones

Resultados

Ocultación

Calidad

Estudio

Métodos

Participantes

Régimen quimioterapia radioterapia

Intervenciones

Resultados

Ocultación

Calidad

\section{Lin 2006}

Ensayo clínico controlado aleatorizado, grupos paralelos, desarrollado en Taiwán.

Pacientes, proveedores y evaluadores cegados. Se describen los motivos de las perdidas, $3 \%$. Seguimiento entre julio de 2003 y agosto 2004. $\mathrm{N}=100$

Adultos con cáncer de cabeza y cuello

Radioterapia fraccionada de 1,8 a 2 Gy/fracción, 5 veces/semana/7-8 semana. Dosis media total: 66-68Gy

Grupo de estudio: Radioterapia + Zinc

Grupo control: Radioterapia + placebo. Zinc capsulas $25 \mathrm{mg}$ tres veces al día desde el inicio hasta el último día de la radioterapia

Evaluación del grado de mucositis oral semanalmente por medio de la escala del Grupo Radiation Therapy Oncology Group

A - Adecuada

A - Bajo riesgo de sesgo
Estudio

Métodos

Participantes

Régimen quimioterapia radioterapia

Intervenciones

Resultados

Ocultación

Calidad

\section{Bensadoun 1999}

Ensayo clínico controlado aleatorizado, grupos paralelos, desarrollado en Francia. Pacientes y evaluadores cegados. No se presentaron perdidas. Seguimiento entre septiembre de 1994 y marzo de 1998. $\mathrm{N}=30$

Carcinoma de hipofaringe, orofaringe y cavidad oral

Radioterapia externa de 60Co o acelerador lineal de fotones, mayor o igual a 65 Gy

Grupo de estudio: Radioterapia + láser de baja energía He-Ne

Grupo control: Radioterapia + placebo. Láser He-Ne diariamente 5 días/semana/7 semana de radioterapia a una dosis de $2 \mathrm{~J} / \mathrm{cm}^{2}$

Grado medio de mucositis durante la radioterapia. Valoración semanal por 7 semanas por medio de la escala estandarizada de la Organización Mundial de la Salud

A - Adecuada

A - Bajo riesgo de sesgo

\section{Arun Maiya 2006}

Ensayo clínico controlado aleatorizado, grupos paralelos, desarrollado en India. Evaluadores cegados. No se presentaron pérdidas. Seguimiento entre junio de $2003 y$ junio de 2004 . N = 50

Carcinoma en cavidad oral estadios II a IV

Radioterapia fraccionada, dosis total 66Gy en seis semanas

Grupo de estudio: Radioterapia + láser de baja energía He-Ne + analgésicos orales Grupo control: Radioterapia + analgésicos orales + anestésicos locales (solución salina $0,9 \%+$ povidona)

Láser He-Ne diariamente 5 días/semana/6 semanas de radioterapia a dosis de $1,8 \mathrm{~J} / \mathrm{cm}^{2}$.

Valoración del grado de mucositis por medio de la escala estandarizada de la Organización Mundial de la Salud

A - Adecuada

C - Alto riesgo de Sesgo 
Estudio

Vacha 2003

\begin{tabular}{|c|c|}
\hline Estudio & Vacha 2003 \\
\hline Métodos & $\begin{array}{l}\text { Ensayo clínico controlado aleatorizado, grupos paralelos, desarrollado en Alemania. Pacientes, } \\
\text { proveedores y evaluadores no cegados. Se describen los motivos de las pérdidas, } 10,7 \% \text {. } \\
\text { Seguimiento entre septiembre } 2006 \text { y febrero } 2009 . \mathrm{N}=56\end{array}$ \\
\hline Participantes & Tumor maligno de laringe, orofaringe e hipofaringe \\
\hline $\begin{array}{l}\text { Régimen quimioterapia - } \\
\text { radioterapia }\end{array}$ & Radioterapia fraccionada convencional. Carboplatin $70 \mathrm{mg} / \mathrm{m} 2 \mathrm{IV}$ \\
\hline Intervenciones & $\begin{array}{l}\text { Grupo de estudio: Carboplatino + radioterapia fraccionada estándar + Amifostine } \\
250 \mathrm{mg} / \mathrm{m}^{2} / \text { día IV } \\
\text { Grupo de control: Carboplatino + radioterapia fraccionada estándar }\end{array}$ \\
\hline Resultados & $\begin{array}{l}\text { Incidencia de mucositis de cualquier grado mediante la escala del Radiation Therapy } \\
\text { Oncology Group }\end{array}$ \\
\hline Ocultación & A - Adecuada \\
\hline Calidad & $\mathrm{C}-$ Alto riesgo de sesgo \\
\hline
\end{tabular}

Métodos

Ensayo clínico controlado aleatorizado, grupos paralelos, pareado por edad, sexo, localización primaria tumoral y dosis de radioterapia. Desarrollado en Irán. Evaluadores cegados. Se describen los motivos de las pérdidas, 10\%. Seguimiento entre marzo 2003 y agosto 2004. $\mathrm{N}=40$

Participantes

Régimen quimioterapia radioterapia

Tumores malignos de nasofaringe, cavidad oral, glándulas salivales, hipofaringe y amígdalas.

Radioterapia 1,8 - 2Gy/día, cinco días por semana. Dosis total: 50 - 60Gy

Grupo de estudio: $20 \mathrm{ml}$ de miel pura natural 15 minutos antes de la radioterapia, 15 minutos después y 6 horas después de la radioterapia. Grupo control: Enjuague de solución salina normal (20 $\mathrm{ml}$ al 0,09\%) antes y después de cada sesión de radioterapia

Intervenciones

Valoración del grado de mucositis mediante Oral Mucositis Assessment Scale al inicio del tratamiento y semanalmente

Resultados

B - Poco claro

Ocultación

B - Moderado riesgo de sesgo

Calidad

Estudio

Biswal 2003

Ensayo clínico controlado aleatorizado, grupos paralelos, desarrollado en Malasia.

Métodos Pacientes, proveedores y evaluadores no cegados. No se presentaron pérdidas. Seguimiento entre noviembre 2000 y octubre 2001. $N=40$

Participantes

Régimen quimioterapia radioterapia

Pacientes con tumores de la cabeza y el cuello

Radioterapia 1,8 - 2Gy/día, cinco días por semana. Dosis total: 50 - 60Gy

Grupo de estudio: Radioterapia + miel

Grupo control: Radioterapia solamente

Intervenciones

Miel, buches y posterior deglución de $20 \mathrm{ml}$ antes, inmediatamente después y a las seis horas de todos los cursos de radioterapia

Cualquier grado de mucositis, mucositis $3 / 4$, grado medio de mucositis, media del

Resultados inicio de mucositis (semanas) y media de la duración total de mucositis (días) valorada semanalmente mediante la escala del Radiation Therapy Oncology Group

$\begin{array}{cl}\text { Ocultación } & \mathrm{A}-\text { Adecuado } \\ \text { Calidad } & \mathrm{C} \text { - Alto riesgo de sesgo }\end{array}$


Métodos

Participantes

Régimen quimioterapia radioterapia

Intervenciones

Resultados

Ocultación

Calidad

Ensayo clínico controlado aleatorizado, grupos paralelos, desarrollado en Holanda. Pacientes, proveedores y evaluadores cegados. Se describieron los motivos de las pérdidas, $10 \%$. Seguimiento entre enero de 1994 y febrero de 1997. $\mathrm{N}=65$

Pacientes con tumor maligno de cabeza y cuello

Radioterapia fraccionada con acelerador lineal de 4-6 MV a una dosis de 2 Gy/fracción, cinco fracciones/semana. Dosis total $50 \mathrm{~Gy}$

Grupo de estudio: Radioterapia + Lozenges

Grupo control: Radioterapia + placebo

Lozenges $1 \mathrm{~g}$ cuatro veces al día (Polimixina E 2mg, Tobramicina 1,8mg y Anfotericina B 10mg)

Incidencia de mucositis grado 3 y 4 medida en dos oportunidades semanalmente y promediadas por medio de la escala estandarizada de la Organización Mundial de la Salud.

A - Adecuado

A - Bajo riesgo de sesgo

Estudio

El-Sayed 2002

Métodos

Ensayo clínico controlado aleatorizado, grupos paralelos, desarrollado en Canadá. Pacientes y proveedores cegados. Se describieron los motivos de las pérdidas, 3,6\%. Seguimiento entre septiembre de 1997 y septiembre de 1999. $\mathrm{N}=138$

Participantes

Pacientes con carcinoma en cavidad oral, faringe o laringe

Régimen quimioterapia radioterapia

$7 \%$ de los pacientes en el grupo de estudio recibieron una dosis total de radioterapia menor o igual a 55Gy, mientras que $10 \%$ del grupo control recibió dicha dosis

Grupo de estudio: Radioterapia + BCoG Lozenge

Intervenciones

Grupo control: Radioterapia + placebo.

BCoG Lozenge (Bacitracina 6mg, Clotrimazol 10mg y Gentamicina 4mg)

Resultados

Tiempo desde el inicio de radioterapia hasta el desarrollo de mucositis grado 2 o 3 (ulceración/ pseudomembranas) mediante Oral Mucositis Assessment Scale

Ocultación

A - Adecuado

Calidad

A - Bajo riesgo de sesgo

Estudio

Etiz 2000

Métodos

Ensayo clínico controlado aleatorizado, grupos paralelos, desarrollado en Turquía. Pacientes y proveedores cegados. No se presentaron pérdidas. Seguimiento entre diciembre de 1996 y diciembre de 1997. $\mathrm{N}=44$

Participantes

Pacientes con tumores malignos de cabeza y cuello

Régimen quimioterapia radioterapia

Radioterapia fraccionada, dosis total 60 a $70 \mathrm{~Gy}$

Grupo de estudio: Radioterapia fraccionada + Sucralfate

Intervenciones

Grupo control: Radioterapia fraccionada + placebo

Sucralfate suspensión oral $6 \mathrm{~g}$ diarios desde el inicio hasta el final de la radioterapia

Resultados

Mediana del grado de mucositis oral por medio de la escala Van der Schueren

Ocultación

A - Adecuado

Calidad

A - Bajo riesgo de sesgo 
Estudio

Métodos

Participantes

Régimen quimioterapia radioterapia

Intervenciones

Resultados

Ocultación

Calidad

\section{Cengiz 1999}

Ensayo clínico controlado aleatorizado, grupos paralelos, desarrollado en Turquía. Pacientes y proveedores cegados. No se presentaron pérdidas. $\mathrm{N}=28$

Pacientes con tumores malignos de cabeza y cuello

Radioterapia fraccionada convencional mediante acelerador lineal 6-MV o teleterapia 60Co. Dosis total: 40 - $70 \mathrm{~Gy}$

Grupo de estudio: Radioterapia fraccionada convencional + Sucralfate. Grupo control: Radioterapia fraccionada convencional + placebo. Sucralfate suspensión enjuague bucal 4 dosis diarias antes de las comidas y al acostarse desde el inicio hasta el final de la radioterapia

Evaluación del grado de mucositis oral dos veces por semana por medio de la escala del Radiation Therapy Oncology Group

A - Adecuado

A - Bajo riesgo de sesgo

\section{Estudio}

Métodos

Participantes

Régimen quimioterapia radioterapia

Intervenciones

Resultados

Ocultación

Calidad

\section{Cerchietti 2006}

Ensayo clínico controlado aleatorizado, grupos paralelos, desarrollado en Argentina. Pacientes y proveedores cegados. Se describen los motivos de las pérdidas. $\mathrm{N}=32$

Pacientes con carcinoma escamocelular de cabeza y cuello

Cisplatino $10 \mathrm{mg} / \mathrm{m} 2$ los días $1^{\circ}$ y $21^{\circ}, 5 \mathrm{FU} 1000 \mathrm{mg} / \mathrm{m}^{2}$ del $1^{\circ}$ al $5^{\circ}$ día y del $21^{\circ}$ al $25^{\circ}$ día. Los días $35^{\circ}, 42^{\circ}, 49^{\circ}$ y $56^{\circ}$ cisplatino $30 \mathrm{mg} / \mathrm{m}^{2}$ seguido de $5 \mathrm{FU} 300 \mathrm{mg} / \mathrm{m}^{2}$. Radioterapia fraccionada iniciada el día $28^{\circ}$ por cinco semanas. Dosis total $70 \mathrm{~Gy}$

Grupo de estudio: Radioterapia + quimioterapia + L-Alanyl-L-Glutamina. Grupo control: Radioterapia + quimioterapia + placebo. L-Alanyl-L-Glutamina 0,4 g/Kg IV

Media de las tres puntuaciones más altas de mucositis medida a través de la escala Objective Mucositis Score y el grado más alto de mucositis según la escala de la Organización Mundial de la Salud

B - Poco clara

B - Moderado riesgo de sesgo
Estudio

Participantes

Régimen quimioterapia radioterapia

Intervenciones

Resultados

Ocultación

Calidad

\section{Madan 2008}

Ensayo clínico controlado aleatorizado, grupos paralelos, pareado por edad, sexo, estadío y localización tumoral. Desarrollado en India. Pacientes y proveedores cegados. No se describen los motivos de las pérdidas, éstas fueron el 10\%. Seguimiento de junio de 2003 a enero de 2004 . $\mathrm{N}=80$

Pacientes con tumores malignos de cabeza y cuello estadios III y IV

Radioterapia fraccionada dosis total 60Gy en seis semanas

Grupo de estudio 1: Radioterapia + clorhexidina 0,12\%. Grupo de estudio 2: Radioterapia + yodopovidona 1\%. Grupo de estudio 3: radioterapia más sal/bicarbonato de sodio. Grupo control: Radioterapia + placebo. Cada solución fue administrada en enjuague bucal $10 \mathrm{ml}$ dos veces al día.

Media del grado de mucositis, medida semanalmente por seis semanas mediante la escala de la Organización Mundial de la Salud

A - Adecuada

$\mathrm{C}$ - Alto riesgo de sesgo 
Estudio

Métodos

Participantes

Régimen quimioterapia radioterapia

Intervenciones

Resultados

Ocultación

Calidad

Estudio

\begin{tabular}{|c|c|}
\hline Estudio & Huang 2000 \\
\hline Métodos & $\begin{array}{l}\text { Ensayo clínico controlado aleatorizado, grupos paralelos. Desarrollado en Taiwán. De julio de } \\
1997 \text { a junio de } 1998 \text {. Sólo los pacientes fueron cegados. No se presentaron pérdidas. } \mathrm{N}=17\end{array}$ \\
\hline Participantes & Pacientes con tumores malignos de cabeza y cuello \\
\hline $\begin{array}{l}\text { Régimen quimioterapia - } \\
\text { radioterapia }\end{array}$ & Radioterapia fraccionada 5 días a la semana hasta completar una dosis total de $45 \mathrm{~Gy}$. \\
\hline Intervenciones & $\begin{array}{l}\text { Grupo de estudio: Radioterapia + Glutamina. Grupo control: Radioterapia + placebo. Glutamina } \\
2 \mathrm{~g} \text { en } 30 \mathrm{ml} \text { de solución salina administrado como enjuague bucal antes de las comidas y al } \\
\text { acostarse }\end{array}$ \\
\hline Resultados & $\begin{array}{l}\text { Media del grado de mucositis a lo largo de la radioterapia medida a través de la escala del } \\
\text { Radiation Therapy Oncology Group }\end{array}$ \\
\hline Ocultación & B - Poco claro \\
\hline Calidad & $\mathrm{C}$ - Alto riesgo de sesgo \\
\hline
\end{tabular}

Ensayo clínico controlado aleatorizado, grupos paralelos. Desarrollado en Tailandia. Sólo los evaluadores fueron cegados. No se presentaron pérdidas. $\mathrm{N}=60$

Pacientes con tumores malignos de cabeza y cuello quienes recibieron radioterapia con o sin otro tratamiento

Radioterapia fraccionada 5 días a la semana hasta completar una dosis total de 60Gy

Grupo de estudio: Radioterapia + glicerina payayor. Grupo control: radioterapia + Benzydamina. Glicerina payayor 2 gotas sobre las lesiones de mucositis 3 a 5 veces al día. Benzydamina enjuague bucal $15 \mathrm{ml}$ tres veces al día

Tiempo medio en semanas de aparición de mucositis. Duración de mucositis oral. Media de severidad del grado de mucositis mediante la escala de la Organización Mundial de la Salud

B - Poco claro

$\mathrm{C}$ - Alto riesgo de sesgo

Estudio

Métodos

Participantes

Régimen quimioterapia radioterapia

Intervenciones

Resultados

Ocultación

Calidad

\section{Veness 2006}

Ensayo clínico controlado aleatorizado, grupos paralelos. Desarrollado en Australia. De 1999 a 2002. Pacientes, proveedores y evaluadores cegados. No se describen pérdidas. $\mathrm{N}=83$

Pacientes con carcinoma escamocelular de cabeza y cuello

Radioterapia fraccionada dosis media 63,8Gy en el grupo de estudio y 62,2Gy en el grupo control. Quimioterapia concomitante: 16 pacientes en el grupo de estudio y 15 en el grupo control

Grupo de estudio: Radioterapia + Misoprostol. Grupo control: Radioterapia + placebo. Misoprostol tabletas de 200 microgramos disueltos en $15 \mathrm{ml}$ de agua administrado diariamente como enjuague bucal y deglución posterior dos horas antes de la radioterapia

Incidencia de mucositis grado 2 y 3 medida mediante la escala del Radiation Therapy Oncology Group

A - Adecuada

$\mathrm{C}$ - Alto riesgo de sesgo 
Estudio

\section{Kin-Fong 2006}

\begin{tabular}{|c|c|}
\hline Métodos & $\begin{array}{l}\text { Ensayo clínico controlado aleatorizado, grupos paralelos. Estudio piloto. Estratificado por } \\
\text { cáncer nasofaríngeo y de otras localizaciones en cabeza y cuello. Desarrollado en China. } \\
\text { Pacientes, proveedores y evaluadores cegados. No se presentaron pérdidas. } \mathrm{N}=14\end{array}$ \\
\hline Participantes & Pacientes con carcinoma escamocelular de cabeza y cuello \\
\hline $\begin{array}{l}\text { Régimen quimioterapia - } \\
\text { radioterapia }\end{array}$ & Radioterapia fraccionada 5 veces por semana a dosis total entre 66 y 68Gy en 7 semanas \\
\hline Intervenciones & $\begin{array}{l}\text { Grupo de estudio: Radioterapia + clorhexidina. Grupo control: Radioterapia más Benzydamine. } \\
\text { Tanto la clorhexidina como el benzydamine fueron administrados en forma de enjuagues } \\
\text { bucales en la mañana y al acostarse desde el primer día de radioterapia hasta dos semanas } \\
\text { posterior a su culminación. }\end{array}$ \\
\hline Resultados & $\begin{array}{l}\text { Incidencia y severidad de la mucositis medida mediante la escala de la Organización Mundial } \\
\text { de la Salud. Intensidad del dolor y disfagia }\end{array}$ \\
\hline Ocultación & A - Adecuada \\
\hline Calidad & A - Bajo riesgo de sesgo \\
\hline Estudio & Wu 2007 \\
\hline Métodos & $\begin{array}{l}\text { Ensayo clínico controlado aleatorizado, grupos paralelos. Desarrollado en China. No se realizó } \\
\text { cegamiento. No se presentaron pérdidas. De febrero de } 2002 \text { a febrero de } 2006 \text {. } N=60\end{array}$ \\
\hline Participantes & Pacientes con tumores malignos de cabeza y cuello \\
\hline $\begin{array}{l}\text { Régimen quimioterapia - } \\
\text { radioterapia }\end{array}$ & Radioterapia fraccionada convencional con media de dosis total de 60Gy \\
\hline Intervenciones & $\begin{array}{l}\text { Grupo de estudio: Radioterapia más QRLYD. Grupo control: Radioterapia más solución de } \\
\text { Dobell's. QRLYD (Flos Lonicerae } 15 \mathrm{~g} \text {, Rhizoma Belamcandae } 15 \mathrm{~g} \text {, Lasiosphaera seu Calvatia } \\
\text { 9g, Radix Astragali 30g, Radix Glehniae 30g, Radix Ophiopogonis 30g, Radix Trichosanthes } 15 \mathrm{~g} \text {, } \\
\text { Radix Scrophulariae 15g, Rhizoma Ligusticum wallichii 15g, Herba Agrimoniae } 20 \mathrm{~g} \text {, Rhizoma } \\
\text { Imperatae 9g, y Radix Glycyrrhizae } 10 \mathrm{~g} \text { ). Doscientos ml vía oral día a lo largo de la radioterapia. } \\
\text { Solución de Dobell's enjuague bucal y posterior deglución } 5 \text { a } 8 \text { veces al día durante el esquema } \\
\text { de radioterapia }\end{array}$ \\
\hline Resultados & $\begin{array}{l}\text { Grado de mucositis al terminar el tratamiento mediante la escala del Radiation Therapy } \\
\text { Oncology Group }\end{array}$ \\
\hline Ocultación & A - Adecuada \\
\hline Calidad & $\mathrm{C}$ - Alto riesgo de sesgo \\
\hline
\end{tabular}

De los 46 ensayos elegibles, 13 no se obtuvieron en texto completo y 11 fueron excluidos por las siguientes razones:

- Inicio de la intervención antes del primer día de quimioterapia, radioterapia o ambas (cinco ensayos: Dörr 2007, Epstein 2002, Grötz 2001, Arora 2008, Su 2006).
- Tipos de medidas de resultado diferentes al que se definió para esta característica de los ensayos (tres ensayos: Duncan 2005, Warde 2002, Chauhan 2008).

- Eligieron niños dentro de los participantes y localizaciones tumorales diferentes a la cabeza y el cuello (dos ensayos: Giles 2003, Giles 2004). 
- Localización tumoral diferente a la cabeza y el cuello (un ensayo: Strasser 2008).

Características de los participantes: De los ensayos incluidos, 14 (63\%) describen que sus participantes son mayores de 18 años, un estudio (Biswal 2003) incluyó un paciente de 14 años con mediana de 54 años para un grupo y 63 para el otro. Ocho ensayos (Brizel 2000, Ertekin 2004, Buentzel 2006, Bensadoun 1999, Cerchietti 2006, Putwatana 2009, Veness 2006, Wu 2007) proporcionan los rangos de edad de sus participantes (mínimo a 18 años y un valor máximo a 82). En ocho experimentos no hay información suficiente que descarte la inclusión de participantes pediátricos; pero la media de edad en cada estudio es de 50 años.

En siete artículos (32\%) el tipo de cáncer por el que se trató a los pacientes fue carcinoma escamocelular de cualquier tejido en cabeza y cue$1 l o$ (Antonadou 2002, Bourhis 2000, Brizel 2000, Buentzel 2006, Cerchietti 2006, Veness 2006, Kin Fong 2006). En tres experimentos (Lin 2006, Etiz 2000, Stokman 2003) la histología fue una combinación de carcinoma escamocelular y no escamocelular. Cuatro estudios (18\%) incluyeron pacientes con primarios desconocidos metastásicos a cabeza y cuello (Brizel 2000, Buentzel 2006, Ertekin 2004, Stokman 2003). En dos ensayos (Ertekin 2004, Wu 2007) algunos de los participantes padecían linfoma ganglionar cervical.

Todos los estudios incluidos en esta revisión incluyeron pacientes sometidos a radioterapia, $13(60 \%)$ sólo radioterapia tres (Buentzel 2006, Vacha 2003, Cerchietti 2006) quimioterapia concomitante en todos los participantes. En cinco estudios solo algunos de los participantes recibieron quimioterapia. Un experimento incluyó participantes que recibieron algún régimen de quimioterapia antes de iniciar el estudio (Cengiz 1999). El régimen de quimioterapia incluyó Carboplatino en dos estudios (Buentzel 2006, Vacha 2003), Cisplatino más 5-fluorouracilo (5-FU) en un ensayo (Cerchietti 2006) y no estaba descrito en el resto de experimentos con algún régimen de quimioterapia.

La radioterapia total para el cáncer de cabeza y cuello fue de 40 a 74 Gy y el rendimiento según la escala de Karnofsky fue mayor de $60 \%$ en cuatro estudios, mayor de $70 \%$ en cuatro ensayos y en 14 experimentos (63\%) no se proporcionan valores de la escala entre sus participantes.
Características de las intervenciones: Los 22 estudios proporcionaron una clara descripción de las intervenciones, que incluyó la dosis y el método de administración tanto en el grupo de estudio como en el grupo control. 12 ensayos (54\%) usaron placebo (Buentzel 2006, Ertekin 2004, Lin 2006, Bensadoun 1999, Stokman 2003, El-Sayed 2002, Etiz 2000, Cengiz 1999, Cerchietti 2006, Madan 2008, Huang 2000, Veness 2006), cinco experimentos $(23 \%)$ no usaron ninguna intervención en el grupo control. Un estudio comparó Clorhexidina en el grupo de prueba, contra Benzinamida en el grupo control, administrándose los fármacos en la misma presentación y posología en ambos grupos (Kin-Fong 2006). En tres ensayos se administró en el grupo control intervenciones activas en presentación y posología distintas a las proporcionadas en el grupo de estudio (Arun Maiya 2006, Putwatana 2009, Wu 2007).

Características de las medidas de resultado: Todos los ensayos emplearon una escala jerarquizada para valorar la severidad de la mucositis oral. El $54 \%$ de los estudios (Antonadou 2002, Bourhis 2000, Brizel 2000, Buentzel 2006, Ertekin 2004, Lin 2006, Vacha 2003, Biswal 2003, Cengiz 1999, Huang 2000, Veness 2006, Wu 2007) emplearon la escala RTOG. Siete experimentos (32\%) evaluaron el grado de mucositis acorde a la escala de la Organización mundial de la Salud (OMS). Dos ensayos usaron la Escala de Evaluación para la Mucositis Oral (OMAS) y en un estudio se empleó la clasificación de Van der Schueren (Etiz 1999). La escala RTOG establece la existencia de 5 grados de mucositis en función de la intensidad de la afectación, desde la no presencia de cambios con respecto a la situación basal (Grado 0) hasta la aparición de ulceración y/o necrosis (Grado 4). La escala de la OMS, además, de establecer la intensidad de la afectación incluye la valoración de la capacidad de deglución del paciente que varía entre 0 (normal) y 4 (grave).

En todos los estudios la valoración de la mucositis se realizó antes y hasta dos semanas de finalización del tratamiento. Los 22 experimentos incluidos valoraron la severidad de la mucositis al menos una vez por semana durante la radioterapia.

\section{Calidad metodológica}

La valoración de la calidad de los estudios según los criterios de los métodos de esta revisión se mostró altamente concordante entre los revisores (Tabla 4). 
Tabla 4. Evaluación de la calidad metodológica de los estudios.

\begin{tabular}{|c|c|c|c|c|c|}
\hline Estudio & Ocultamiento & $\begin{array}{c}\text { Desenlace } \\
\text { cegado }\end{array}$ & $\begin{array}{l}\text { Descripción } \\
\text { perdidas }\end{array}$ & $\begin{array}{l}\text { Riesgo de } \\
\text { sesgo }\end{array}$ & $\begin{array}{c}\text { Pérdidas } \\
\text { (\%) }\end{array}$ \\
\hline Antonadou 2002 & A & No cumple & $\mathrm{Si}$ & Alto & 10 \\
\hline Arun Maiya 2006 & A & No cumple & $\mathrm{Si}$ & Alto & 0 \\
\hline Bensadoun 1999 & A & Cumple & $\mathrm{Si}$ & Bajo & 0 \\
\hline Biswal 2003 & A & No cumple & $\mathrm{Si}$ & Alto & 0 \\
\hline Bourhis 2000 & A & No cumple & $\mathrm{Si}$ & Alto & 7,7 \\
\hline Brizel 2000 & A & No cumple & $\mathrm{Si}$ & Alto & 4 \\
\hline Buentzel 2006 & A & Cumple & $\mathrm{Si}$ & Bajo & 22,7 \\
\hline Cengiz 1999 & A & Cumple & $\mathrm{Si}$ & Bajo & 0 \\
\hline Cerchietti 2006 & B & Cumple & $\mathrm{Si}$ & Moderado & 9,3 \\
\hline El-Sayed 2002 & A & Cumple & $\mathrm{Si}$ & Bajo & 3,6 \\
\hline Ertekin 2004 & A & Cumple & $\mathrm{Si}$ & Bajo & 10 \\
\hline Etiz 2000 & A & Cumple & $\mathrm{Si}$ & Bajo & 0 \\
\hline Huang 2000 & B & No cumple & $\mathrm{Si}$ & Alto & 0 \\
\hline Kin-Fong 2006 & A & Cumple & $\mathrm{Si}$ & Bajo & 0 \\
\hline Lin 2006 & A & Cumple & $\mathrm{Si}$ & Bajo & 3 \\
\hline Madan 2008 & A & Cumple & No & Alto & 10 \\
\hline Motallebnejad 2008 & B & Inadecuado & $\mathrm{Si}$ & Moderado & 10 \\
\hline Putwatana 2009 & B & No cumple & $\mathrm{Si}$ & Alto & 0 \\
\hline Stokman 2003 & A & Cumple & $\mathrm{Si}$ & Bajo & 10 \\
\hline Vacha 2003 & A & No cumple & $\mathrm{Si}$ & Alto & 10,7 \\
\hline Veness 2006 & A & Cumple & No & Alto & Incierto \\
\hline Wu 2007 & A & No cumple & $\mathrm{Si}$ & Moderado & 0 \\
\hline
\end{tabular}

\section{Metanálisis}

La comparación de las intervenciones se realizó por grupos, así:

- Amifostine vs. placebo/ningún tratamiento: Cinco estudios compararon amifostine con placebo/ ningún tratamiento. Cuatro estudios (Antonadou 2002, Bourhis 2000, Brizel 2000, Buentzel 2006) proporcionaron datos de mucositis grado 3 o mayor de acuerdo con la escala RTOG; dada la heterogeneidad se analizaron los resultados de los estudios con el método de efectos aleatorios. No hubo beneficio significativo para prevenir la mucositis con amifostine (Gráfica 1). Adicionalmente un estudio realizado por Vacha en el 2003 mostró beneficio significativo en la reducción de la mucositis durante la segunda semana de tratamiento con amifostine $(p=0.05)$.
- Láser He-Ne vs. placebo/ninguna intervención: Dos estudios (Bensadoun 1999, Arun Maiya 2006), compararon láser He-Ne con placebo o ninguna intervención. Ambos ensayos proporcionaron datos de la media del grado de mucositis según la escala RTOG; dada la heterogeneidad se analizaron los resultados de los estudios con el método de efectos aleatorios y mostraron que no hubo beneficio significativo para prevenir la mucositis con láser He-Ne (Gráfica 2).

- Zinc vs. placebo: dos estudios, Ertekin 2004 y Lin 2006, compararon Zinc vs. placebo. Los estudios fueron homogéneos entre si, por lo que se uso el método de efectos fijos. El riesgo de tener mucositis grado 2 o grado 3 según la escala RTOG al final del tratamiento con radioterapia fue menor en el grupo de estudio para ambos casos (Gráfica 3, 4). 


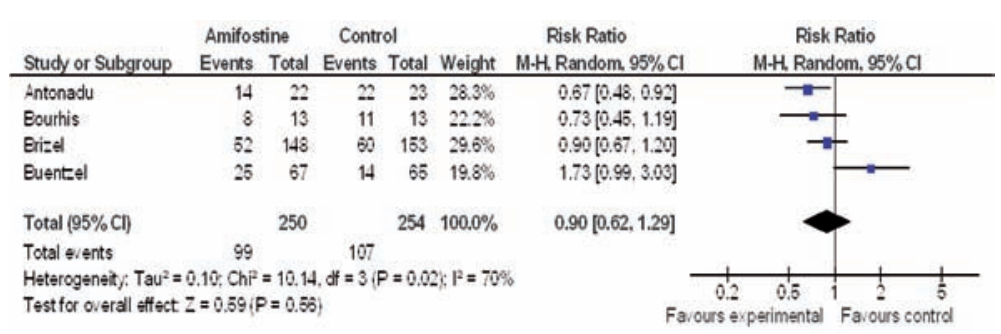

Gráfica 1. Beneficio de la intervención con Amifostine para la prevención de la mucositis oral segundaria a quimioterapia, radioterapia o ambas en pacientes con cáncer de cabeza y cuello.

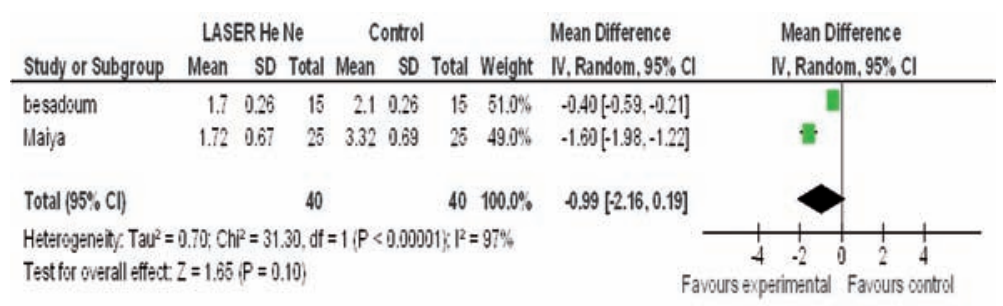

Gráfica 2. Beneficio de la intervención con Laser He-Ne para la prevención de la mucositis oral segundaria a quimioterapia, radioterapia o ambas en pacientes con cáncer de cabeza y cuello.

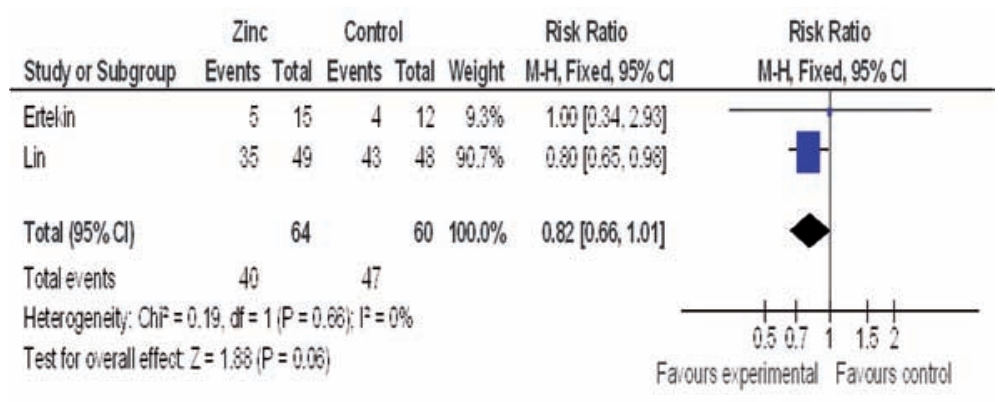

Gráfica 3. Beneficio de la intervención con Zinc para la prevención de la mucositis oral grado 2 secundaria a quimioterapia, radioterapia o ambas en pacientes con cáncer de cabeza y cuello.

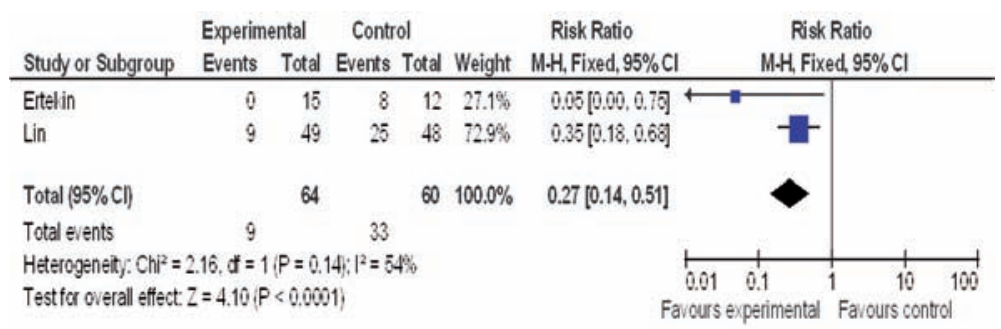

Gráfica 4. Beneficio de la intervención con Zinc para la prevención de la mucositis oral grado 3 segundaria a quimioterapia, radioterapia o ambas en pacientes con cáncer de cabeza y cuello.

\section{DISCUSIÓN}

Los experimentos clínicos publicados en la literatura médica que evalúan estrategias de prevención para la mucositis oral secundaria a radioterapia, quimioterapia o ambas muestran una heterogeneidad de intervenciones que señala la falta de una terapia eficaz y la necesidad clínica existente en torno a esta toxi- cidad de la terapia contra el cáncer. La estrategia de búsqueda de esta revisión identificó diferentes ensayos clínicos aleatorizados que evaluaron 15 intervenciones distintas de prevención de la mucositis en los últimos 10 años. Los múltiples estudios con distintas intervenciones para el manejo de la mucositis son consecuencia de la falta de un manejo estandarizado para prevenir esta complicación. 
Dada la variedad de terapias preventivas comparadas en los ensayos clínicos incluidos, solo tres (amifostine, zinc, láser $\mathrm{He} / \mathrm{Ne}$ ) aportaron experimentos que permitieron la realización de metanálisis. La existencia de pocos estudios que investiguen las mismas intervenciones limita la fuerza de la evidencia y la posibilidad de generalizar los resultados. Ninguno de los 22 experimentos reportó que la intervención en el grupo de estudio representara un riesgo significativo de daño entre los participantes. Sólo fue posible detectar sesgo de publicación para los estudios que compararon amifostine vs. placebo, se presume que se han perdido estudios pequeños que muestran un resultado negativo para este tipo de comparación. De las 15 intervenciones nueve presentaron prueba de algún beneficio para prevenir o reducir la gravedad de la mucositis.

Múltiples estudios se han realizado para evaluar la efectividad del amifostine. En dos metanálisis de prevención de la mucositis asociada a radioterapia en cabeza y cuello se demostró un pequeño beneficio del amifostine para prevenir la mucositis y disminución de la severidad de la misma. ${ }^{10-13} \mathrm{En}$ la revisión actual se incluyeron cinco estudios que evaluaron el amifostine, Vacha en el año 2003 demostró beneficio en la reducción de la mucositis durante la segunda semana de tratamiento con amifostine,,$^{14}$ este ensayo se consideró con alto riesgo de sesgo y no presentaba datos comparables que permitieran su inclusión en el metanálisis. Por otro lado, el metanálisis de los cuatro estudios restantes que evaluaron amifostine contra placebo o ninguna intervención, no se demostró beneficio significativo en la reducción de la severidad de mucositis.

En cuanto al láser He-Ne a pesar de que los estudios han demostrado beneficio en el control del dolor y en la cicatrización (Bensadou 1999, Arun 2006, Arora 2008), en el metanálisis realizado no se observó beneficio significativo para prevenir la mucositis. Las sustancias y técnicas descritas no han demostrado efectos nocivos por su aplicación a los pacientes, excepto, el uso de láser que puede producir lesiones en la retina si no se tiene en cuenta la adecuada protección. ${ }^{15-17}$

El Zinc es un nutriente con una función bioquímica importante en el mantenimiento de la estructura y función de la membrana celular y en el metabolismo de la piel, el tejido conectivo y en la recuperación de las heridas. ${ }^{18}$ Dos estudios considerados con bajo riesgo de sesgo evaluaron el Zinc vía oral vs. placebo y encontraron beneficio significativo en disminuir la severidad de la mucositis. El metanálisis realizado mostró que el riesgo de desarrollar mucositis grado 2 en los pacientes que recibieron zinc es
$20 \%$ menor que en el grupo control, demuestra ser útil para estos pacientes.

Por otro lado, el sucralfate y la miel a pesar de no poder llevarse a metanálisis por no presentar resultados comparables mostraron beneficio en el manejo de la mucositis en los estudios hechos por Etiz y colaboradores (2000), y Motallebnejad y colaboradores (2008). ${ }^{19,20}$

Los ensayos clínicos con las intervenciones mencionadas han arrojado resultados inconsistentes, de modo que ninguno de ellos se ha convertido en un complemento con probada efectividad en la terapia de la mucositis oral segundaria a quimioterapia, radioterapia o ambas en pacientes con cáncer de cabeza y cuello, excepto, el zinc que mostró una reducción estadísticamente significativa en el grado de mucositis oral. De tal manera, es necesario continuar con nuevas investigaciones para encontrar la intervención preventiva que genere mayores beneficios para el paciente.

\section{REFERENCIAS}

1. Fang L Sonis ST, Fazio RC. Complicações bucais da quimioterapia do câncer. Medicina Oral. Rio de Janeiro: Guanabara Koogan, 1989.

2. Puyal-Casado M, Jimenez-Martinez C, Chimenos-Kustner E, Lopez-Lopez J. A protocol for the evaluation and treatment of oral mucositis in patients with hematological malignancies. Med Oral 2003; 8: 10 - 18.

3. Adamietz IA, Rahn R, Böttcher HD, Schäfer V, Reimer K, Fleischer W. Prophylaxis with povidone-iodine against induction of oral mucositis by radiochemotherapy. Support Care Cancer 1998; 6: 373 - 377.

4. Parulekar W, Mackenzie R, Bjarnason G, Jordan RCK. Scoring oral mucositis. Oral Oncol 1998; 34: 63 - 71.

5. Raber-Durlacher JE. Current practices for management of oral mucositis in cancer patients. Support Care Cancer 1999; 7: 71 - 74.

6. Lalla RV, Peterson DE. Oral mucositis. Dent Clin North Am Jan. 2005; 49: 167 - 184.

7. Trotti A, Bellm LA, Epstein JB, Frame D, Fuchs HJ, Gwede $\mathrm{CK}$, et al. Mucositis incidence, severity and associated outcomes in patients with head and neck cancer receiving radiotherapy with or without chemotherapy: a systematic literature review. Radiother Oncol 2003; 66: 253 - 262.

8. Bensadoun RJ, Magné N, Marcy PY, Demard F. Chemotherapy and radiotherapy-induced mucositis in head and neck cancer patients: new trends in pathophysiology, prevention and treatment. Eur Arch Otorhinolaryngol 2001; 258: 481 - 487.

9. Sonis ST. Mucositis is as a biological process: a new hypothesis for development of chemotherapy-induced stomato-toxicity. Oral Oncol 1998; 34: 39 - 43.

10. Worthington HV, Clarkson JE, Eden OB. Intervenciones para la prevención de la mucositis oral en pacientes que reciben tratamiento para el cáncer (Revisión Cochrane traducida). En: la Biblioteca Cochrane Plus, 2008 Número 2. Oxford: Update Software Ltd.

11. Sasse AD, Clark LG, Sasse EC, Clark OA. Amifostine redu- 
ces side effects and improves complete response rate during radiotherapy: results of a meta-analysis. Int J Radiat Oncol Biol Phys 2006; 64: 784 - 791.

12. Gallego C. La mucositis, un efecto del tratamiento quimioterapéutico: fisiopatología y manejo. Rev Fac Odontol Univ Antioq 2007; 18: 84 - 92.

13. Bagchi D, Bagchi M, Stohs SJ. Comparative in vitro oxygen radical scavenging ability of zinc methionine and selected zinc salts and antioxidants. Gen Pharmacol 1997; 28: $85-91$.

14. Vacha P, Fehlauer F, Mahlmann B, Marx M, Hinke A, Sommer K, et al. Randomized phase III trial of postoperative radiochemotherapy amifostine in head and neck cancer: Is there evidence for radioprotection? Strahlenther Onkol 2003; 179: 385 - 389.

15. Bensadoun RJ, Franquin JC, Ciais G, Darcourt V, Schubert MM, Viot M, et al. Low-energy He/Ne laser in the prevention of radiation-induced mucositis. A multicentre phase III randomized study in patients with head and neck cancer. Support Care Cancer 1999; 7: 244 - 252.

16. Arun Maiya G, Sagar MS, Fernandes D. Effect of low-level helium-neon (He-Ne) laser therapy in the prevention \& treatment of radiation induced mucositis in head \& neck cancer patients. Indian J Med Res 2006; 124: 399 - 402.

17. Arora, H, Pai KM., Maiya A, Vidyasagar MS, Rajeev A. Efficacy of He-Ne Laser in the prevention and treatment of radiotherapy-induced oral mucositis in oral cancer patients. Oral Surg Oral Med Oral Pathol Oral Radiol Endod 2008; 105: 180 - 186.

18. Wadleigh RG, Redman RS, Graham ML, Krasnow SH, Anderson A, Cohen MH. Vitamin E in the treatment of chemotherapy-induced mucositis. Am J Med 1992; 92: 481 - 484.

19. Etiz D, Erkal HS, Serin M, Küçük B, Hepari A, Elhan AH, et al. Clinical and histopathological evaluation of sucralfate in prevention of oral mucositis induced by radiation therapy in patients with head and neck malignancies. Oral Oncol 2000; 36: 116 - 120.

20. Motallebnejad M, Akram S, Moghadamnia A, Moulana Z, Omidi S. The effect of topical application of pure honey on radiation-induced mucositis: a randomized clinical trial. J Contemp Dent Pract 2008; 9: 40 - 47.

Referencias de los estudios incluidos en esta revisión

- $\quad$ Antonadou 2002

Antonadou D, Pepelassi M, Synodinou M, Puglisi M, Throuvalas N. Prophylactic use of amifostine to prevent radiochemotherapy-induced mucositis and xerostomia in head-and-neck cancer. Int J Radiat Oncol Biol Phys 2002; 52: 739 - 747.

- $\quad$ Arun Maiya 2006

Arun Maiya G, Sagar MS, Fernandes D. Effect of low-level helium-neon (He-Ne) laser therapy in the prevention \& treatment of radiation induced mucositis in head \& neck cancer patients. Indian J Med Res 2006; 124: 399 - 402.

- $\quad$ Bensadoun 1999

Bensadoun RJ, Franquin JC, Ciais G, Darcourt V, Schubert $\mathrm{MM}$, Viot M, et al. Low-energy He/Ne laser in the prevention of radiation-induced mucositis. A multicentre phase III randomized study in patients with head and neck cancer. Support Care Cancer 1999; 7: 244 - 252.

- $\quad$ Biswal 2003

Biswal BM, Zakaria A, Ahmad NM. Topical application of honey in the management of radiation mucositis. A preliminary study. Support Care in Cancer 2003; 11: 242 - 248.

- $\quad$ Bourhis 2000
Bourhis J, De Crevoisier R, Abdulkarim B, Deutsch E, Lusinchi A, Luboinski B, et al. A randomized study of very accelerated radiotherapy with and without amifostine in head and neck squamous cell carcinoma. Int J Radiat Oncol Biol Phys 2000; 46: 1105 - 1108.

- $\quad$ Brizel 2000

Brizel DM, Wasserman TH, Henke H, Strnad V, Rudat V, Monnier A, et al. Phase III randomized trial of amifostine as a radioprotector in head and neck cancer. J Clin Oncol 2000; 18: 3339 - 3345.

- $\quad$ Buentzel 2006

Buentzel J, Micke O, Adamietz IA, Monnier A, Glatzel M, de Vries A. Intravenous amifostine during chemoradiotherapy for head-and-neck cancer: a randomized placebo-controlled phase III study. Int J Radiat Oncol Biol Phys 2006; 64: 684 - 691.

- $\quad$ Cengiz 1999

Cengiz M, Ozyar E, Ozturk D, Akyol F, Atahan IL, Hayran M. Sucralfate in the prevention of radiation-induced oral mucositis. J Clin Gastroenterol 1999; 28: 40 - 43.

- $\quad$ Cerchietti 2006

Cerchietti LC, Navigante AH, Lutteral MA, Castro MA, Kirchuk R, Bonomi M, et al. Double-blinded, placebo-controlled trial on intravenous L-alanyl-L-glutamine in the incidence of oral mucositis following chemoradiotherapy in patients with head-and-neck cancer. Int J Radiat Oncol Biol Phys 2006; 65: 1330 - 1337.

- $\quad$ El-Sayed 2002

El-Sayed S, Nabid A, Shelley W, Hay J, Balogh J, Gelinas $\mathrm{M}$, et al. Prophylaxis of radiation-associated mucositis in conventionally treated patients with head and neck cancer: a double-blind, phase III, randomized, controlled trial evaluating the clinical efficacy of an antimicrobial lozenge using a validated mucositis scoring system. J Clin Oncol 2002; 20: 3956 - 3963.

- $\quad$ Ertekin 2004

Ertekin MV, Koc M, Karslioglu I, Sezen O. Zinc sulfate in the prevention of radiation-induced oropharyngeal mucositis: a prospective, placebo-controlled, randomized study. Int J Radiat Oncol Biol Phys 2004; 58: 167 - 174.

- $\quad$ Etiz 2000

Etiz D, Erkal HS, Serin M, Küçük B, Hepari A, Elhan AH, et al. Clinical and histopathological evaluation of sucralfate in prevention of oral mucositis induced by radiation therapy in patients with head and neck malignancies. Oral Oncol 2000; 36: 116 - 120.

- $\quad$ Huang 2000

Huang EY, Leung SW, Wang CJ, Chen HC, Sun LM, Fang FM, et al. Oral glutamine to alleviate radiation-induced oral mucositis: a pilot randomized trial. Int J Radiat Oncol Biol Phys 2000; 46: 535 - 539.

- $\quad$ Kin-Fong 2006

Kin-Fong Cheng K, Ka Tsui Yuen J. A pilot study of chlorhexidine and benzydamine oral rinses for the prevention and treatment of irradiation mucositis in patients with head and neck cancer. Cancer Nurs 2006; 29: 423 - 430.

- $\quad$ Lin 2006

Lin LC, Que J, Lin LK, Lin FC. Zinc supplementation to improve mucositis and dermatitis in patients after radiotherapy for head-and-neck cancers: a double-blind, randomized study. Int J Radiat Oncol Biol Phys 2006; 65: 745-50.

- Madan 2008

Madan PD, Sequeira PS, Shenoy K, Shetty J. The effect of three mouthwashes on radiation-induced oral mucositis in patients with head and neck malignancies: a randomized control trial. J Cancer Res Ther 2008; 4: 3 - 8. 
- $\quad$ Motallebnejad 2008

Motallebnejad M, Akram S, Moghadamnia A, Moulana Z, Omidi S. The effect of topical application of pure honey on radiation-induced mucositis: a randomized clinical trial. J Contemp Dent Pract 2008; 9: 40 - 47.

- Putwatana 2009

Putwatana P, Sanmanowong P, Oonprasertpong L, Junda T, Pitiporn S, Narkwong L. Relief of radiation-induced oral mucositis in head and neck cancer. Cancer Nurs 2009; 32: 82 - 87.

- $\quad$ Stokman 2003

Stokman MA, Spijkervet FK, Burlage FR, Dijkstra PU, Manson WL, de Vries EG, et al. Oral mucositis and selective elimination of oral flora in head and neck cancer patients receiving radiotherapy: a double-blind randomised clinical trial. Br J Cancer 2003; 88: 1012 - 1016.

- Vacha 2003

Vacha P, Fehlauer F, Mahlmann B, Marx M, Hinke A, Sommer $\mathrm{K}$, et al. Randomized phase III trial of postoperative radiochemotherapy amifostine in head and neck cancer: Is there evidence for radioprotection? Strahlenther Onkol 2003; 179: 385 - 389.

- $\quad$ Veness 2006

Veness MJ, Foroudi F, Gebski V, Timms I, Sathiyaseelan Y, Cakir B, et al. Use of topical misoprostol to reduce radiation-induced mucositis: results of a randomized, doubleblind, placebo-controlled trial. Australas Radiol 2006; 50: $468-474$.

- $\quad$ Wu 2007

Wu MH, Yuan B, Liu QF, Wang Q. Study of qingre liyan decoction in treating and preventing acute radioactive oral mucositis. Chin J Integr Med 2007; 13: 280 - 284.

Referencias de los estudios excluidos de esta revisión

- $\quad$ NCI 2008

National Cancer Institute (NCI), Phase III comparison of oral capsaicin lozenge versus placebo lozenge for radiation-induced mucositis [completed] 2008. URL disponible en: www.clinicaltrials.gov ID: NCT00003610.

- $\quad$ Arora 2008

Arora, H, Pai KM., Maiya A, Vidyasagar MS, Rajeev A. Efficacy of He-Ne Laser in the prevention and treatment of radiotherapy-induced oral mucositis in oral cancer patients. Oral Surg Oral Med Oral Pathol Oral Radiol Endod 2008; 105: 180 - 186.

- Braaksma 2002

Braaksma M, Levendag P. Tools for optimal tissue sparing in concomitant chemoradiation of advanced head and neck cancer: subcutaneous amifostine and computed tomography-based target delineation. Semin Oncol 2002; 29: 63 - 70.

- Carter 1999

Carter, DL, M. Hebert E, Smink K, Leopold KA, Clough RL. Brizel DM. Double blind randomized trial of sucralfate vs placebo during radical radiotherapy for head and neck cancers. Head Neck 1999; 21: 760 - 766.

- Chauhan 2008

Chauhan A, Singh H., Sharma T, Manocha K. Gemcitabine concurrent with radiation therapy for locally advanced head and neck carcinomas. Afr Health Sci 2008; 8: 149 - 155.

- $\quad$ Dörr 2007

Dörr W, T. Herrmann. Efficacy of Wobe-Mugos E for reduction of oral mucositis after radiotherapy: results of a prospective, randomized, placebo-controlled, triple-blind phase III multicenter study. Strahlenther Onkol 2007; 183: $121-127$.

\section{- Duncan 2005}

Duncan, G. G., J. B. Epstein, D. Tu, S. E. Sayed, A. Bezjak, J. Ottaway, J. Pater, N. C. I. of Canada Clinical Trials Group 2005. Quality of life, mucositis, and xerostomia from radiotherapy for head and neck cancers: a report from the NCIC CTG HN2 randomized trial of an antimicrobial lozenge to prevent mucositis. Head Neck 2005; 27: 421 $-428$.

- $\quad$ Epstein 2002

Epstein JB, Silverman S, Paggiarino DA, Crockett S, Schubert M., Senzer NN, et al. Benzydamine $\mathrm{HCl}$ for prophylaxis of radiation-induced oral mucositis: results from a multicenter, randomized, double-blind, placebo-controlled clinical trial. Cancer 2002; 92: 875 - 885.

- $\quad$ Ferreira 2004

Ferreira PR, Fleck JF, Diehl A, Barletta D, Braga-Filho A, Barletta A, et al. Protective effect of alpha-tocopherol in head and neck cancer radiation-induced mucositis: a double-blind randomized trial. Head Neck 2004; 26: $313-321$.

- $\quad$ Giles 2003

Giles FJ, Miller CB, Hurd DD, Wingard JR, Fleming TR, Sonis ST, et al. A phase III, randomized, double-blind, placebo-controlled, multinational trial of iseganan for the prevention of oral mucositis in patients receiving stomatotoxic chemotherapy (PROMPT-CT trial). Leuk Lymphoma 2003; 44: 1165 - 1172.

- Giles 2004

Giles FJ, Rodriguez R, Weisdorf D, Wingard JR, Martin PJ, Fleming TR, et al. A phase III, randomized, double-blind, placebo-controlled, study of iseganan for the reduction of stomatitis in patients receiving stomatotoxic chemotherapy. Leuk Res 2004; 28: 559 - 565.

- Grötz 1999

Grötz KA., Von Zepelin HH. Kohnen RB. Al-Nawas A. Bockisch J. Kutzner P, et al. Prospective double-blind study of prophylaxis of radioxerostomia with Coumarin/Troxerutine in patients with head and neck cancer. Strahlenther Onkol 1999; 175: 397 - 403.

- Grötz 2001

Grötz KA. Westenberg A, Kohnen RB. Al-Nawas A, Von Zepelin HH., Bockisch A, et al. Prophylaxis of radiogenic sialadenitis and mucositis by coumarin/troxerutine in patients with head and neck cancer -a prospective,randomized, placebo-controlled, double-blind study. Br J Oral Maxillofac Surg 2001; 39: 34 - 39.

- Hong 2000

Hong Ding Z. Prevention and treatment of oral mucositis caused by radiotherapy in patients with nasopharyngeal carcinoma. Journal of Ningxia Medical College. 2000; 22 (2).

- Penpattanagul 2007

Penpattanagul S. Reduced incidence and severity of acute radiation mucositis by WF10 (IMMUNOKINE) as adjunct to standard of cure in the management of head \& neck cancer patients. J Med Assoc Thai 2007; 90: 1590 - 1600.

- $\quad$ Peters 1999

Peters K, Mücke R, Hamann RD, Ziegler PG, Fietkau R. Supportive use of amifostine in patients with head and neck tumors undergoing radio-chemotherapy. Is it possible to limit the duration of the application of amifostine? Strahlenther Onkol 1999; 175: 23 - 26.

\section{- $\quad$ Rashad 2009}

Rashad UM, Al-Gezawy SM, El-Gezawy E, Azzaz AN. Honey as topical prophylaxis against radiochemotherapy-induced mucositis in head and neck cancer. J Laryngol Otol 2009; 123: 223 - 228. 


\section{- $\quad$ Schneider 1999}

Schneider SB, Nishimura RD, Zimmerman RP, Tran L, Shiplacoff J, Tormey M, et al. Filgrastim (r-metHuG-CSF) and its potential use in the reduction of radiation-induced oropharyngeal mucositis: an interim look at a randomized, double-blind, placebo-controlled trial. Cytokines Cell Mol Ther 1999; 5: 175 - 180.

- $\quad$ Strasser 2008

Strasser F, Demmer R, Bphme C, Schmitz SF, B Thuerlimann., Cerny T, et al. Prevention of docetaxel- or paclitaxel-associated taste alterations in cancer patients with oral glutamine: a randomized, placebo-controlled, double-blind study. Oncologist 2008; 13: 337 - 346.

- $\quad$ Su 2006

Su YB, Vickers AJ, Zelefsky M J, D Kraus H, Shaha AR, Shah JP, et al. Double-blind, placebo-controlled, randomized trial of granulocyte-colony stimulating factor during postoperative radiotherapy for squamous head and neck cancer. Cancer J 2009; 12: 182 - 188.

- Vacha 1999

Vacha P, Marx M, Engel A, Richter E, Feyerabend T. Side effects of postoperative radiochemotherapy with amifostine versus radiochemotherapy alone in head and neck tumors. Preliminary results of a prospective randomized trial. Strahlenther Onkol 1999; 175: 18 - 22.

- Veerasarn 2006

Veerasarn V, Phromratanapongse P, Suntornpong N, Lorvidhaya V, Sukthomya V, Chitapanarux I, et al. Effect of Amifostine to prevent radiotherapy-induced acute and late toxicity in head and neck cancer patients who had normal or mild impaired salivary gland function. J Med Assoc Thai 2006; 89: 2056- 2067.

- $\quad$ Warde 2002

Warde P, O’Sullivan B, Aslanidis J, Kroll B, Lockwood G, Waldron J, et al. A Phase III placebo-controlled trial of oral pilocarpine in patients undergoing radiotherapy for head-and-neck cancer. Int J Radiat Oncol Biol Phys 2002; 54: 9 - 13.

- $\quad$ Shen 2004

Shen YF, Ma BL, Huang H. Effect of vitamin B12 and dexasone on acute radiation stomatitis. Chinese New Medicine. 2004; 5: 509 - 510.

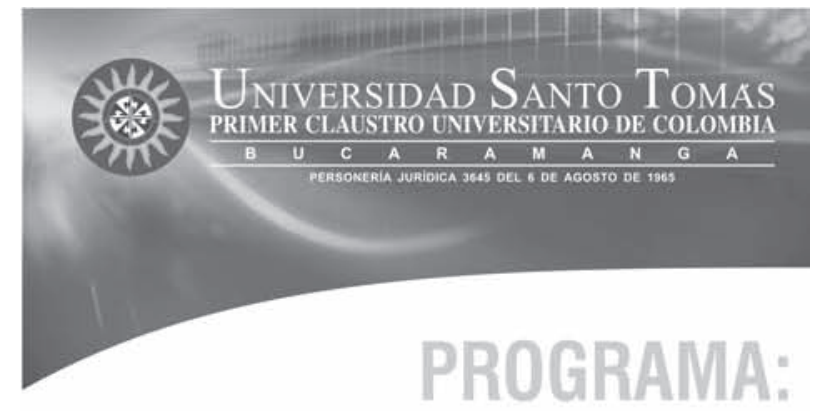

\section{ODONTOLOGÍA}

SNIES 1097

Acreditación de Alta Calidad 517 del 6 de febrero de 2008

www.ustabuca.edu.co

e-mail: mercadeo@mail.ustabuca.edu.co

6800801 Ext. 1801 - 1263 - 1264 - Línea Gratuita 018000917044

\section{Correos electrónicos de los autores:}

Johanna Alejandra Otero Wandurraga: jaoterow@gmail.com, Harold Torres Pinzón: haroldtorpin@gmail.com, Ana María Pedraza Flechas: anipf@yahoo.com, Abel Ernesto González Vélez: aegonzalezv@gmail.com

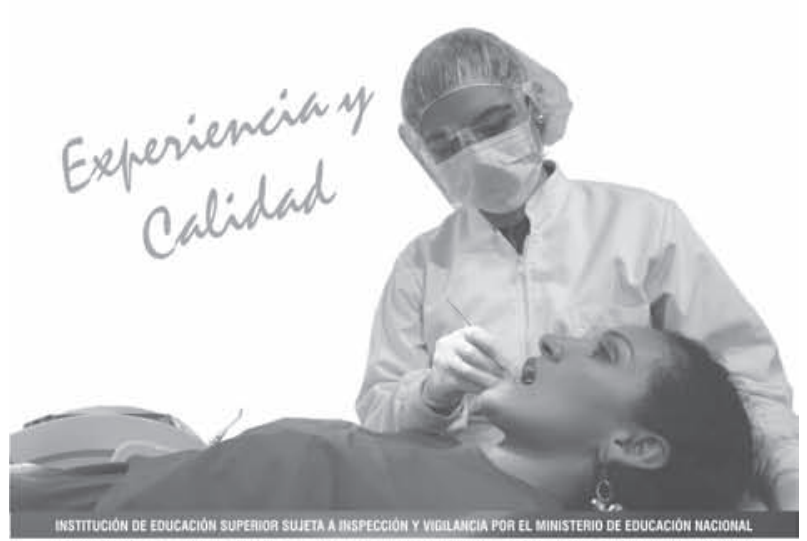

